\title{
LA EVALUACION Y LA UNIVERSIDAD COMO OBJETO DE ESTUDIO
}

\author{
Augusto Perez Lindo* \\ Recebido em: 27/07/07 \\ Avaliado em: 20/09/07 \\ *Ph.D. en Filosofía por la Universidad Católica de Lovaina. Director de la Maestría en Gestión y \\ Políticas Universitarias del Mercosur, Universidad Nacional de Lomas de Zamora (Argentina), \\ Profesor Titular de Filosofía de la UBA. E-mail: perezlindo@arnet.com.ar \\ Resumen: Nos proponemos en este artículo analizar la evolución de los estudios sobre la educación \\ superior y las relaciones con la evaluación institucional universitaria. Interpretamos que \\ la aparición de los estudios sobre la educación superior están relacionados con la \\ emergencia de una sociedad que se produce a través del conocimiento.
}

Palabras clave: Educación superior; evaluación institucional; estudios sobre educación superior; epistemología y evaluación.

\section{Evaluation and the University as Research Objects}

Abstract: In the present article we present an analysis of the evolution of the studies on higher education and their relation with higher education institutional evaluation. We interpret that studies on higher education begin to appear along with the emergence of a society that is produced through knowledge.

Keywords: Higher education; institutional evaluation; studies on higher education; epistemology and evaluation.

\section{Los estudios sobre educación superior y el nacimiento de la evaluación institucional}

Los programas evaluativos actuales tienen que comprenderse como parte de un proceso iniciado a fines de los años 1960 para estudiar y reformar las universidades. No puede desvincularse la teoría y la metodología de la evaluación institucional de los estudios sobre la educación superior. Esto debería ser obvio, pero no lo es. Los criterios de evaluación suelen responder a exigencias del control de gestión, de la evaluación de resulta- 
dos o de procesos. La construcción y el análisis de indicadores están normalmente vinculados a requisitos de carácter institucional.

Como corolario de nuestro análisis sostenemos que las agencias de evaluación deberían deberían tomar en cuenta criterios epistemológicos al mismo tiempo que institucionales. La construcción del objeto de estudio, la universidad, merece el mismo status de objetividad para la investigación y para la evaluación.

\section{La opacidad de la educación superior}

$\mathrm{Al}$ iniciar el estudio de las universidades lo primero que sorprende es su poca transparencia. Jean Debelle (1983, p. 113) manifiesta al respecto:

Qué cosa más desconcertante: esta Universidad que pretende ser el más alto grado de la investigación y del saber, que explora todos los dominios del conocimiento fundamental o aplicado, esta universidad, se encuentra extremadamente desamparada cuando se trata de ella misma.

Burton Clark (1986, p. 1) por su parte hace el siguiente comentario:

Por mucho tiempo los académicos no abordaron seriamente el dominio de su propia competencia. Mientras desarrollaban perspectivas sistemáticas sobre la economía, la política y tales campos como el sistema de clases sociales, solo ocasionalmente se reunían comentarios de rectores o profesores retirados sobre el funcionamiento de los sistemas de educación superior.

Podríamos citar a otros autores que también se sorprenden con la relativa "opacidad" que envuelve el conocimiento de la universidad (por ejemplo: Pierre Bourdieu en el Prefacio de "Homus Academicus"). Partiendo de estos testimonios podemos proponer algunas hipótesis. La primera, que la universidad ha permanecido hasta muy poco tiempo en la penumbra, ha sido muy poco estudiada. Por lo tanto, existe una brecha importante entre su realidad objetiva y lo que se dice de ella.

La segunda hipótesis sería que la opacidad de las instituciones universitarias se debe tanto a la complejidad del objeto o como al ocultamiento de los actores. Cualquier evaluador, cualquier estudioso de la universidad se ha enfrentado con este problema. 
La tercera hipótesis sería que sin una teoría de la universidad es probable que no podamos alcanzar una visión o interpretación objetiva de la universidad. Aquí dejamos pendiente la cuestión de saber si es posible el conocimiento objetivo de la universidad o si se trata de alcanzar una interpretación compartida entre los actores involucrados. En cualquier caso necesitamos una teoría para construir el objeto de observación.

\section{De la consciencia histórica al autonoconocimiento de la educación}

En la Europa del Siglo XIX y XX algunos autores como Hegel, Marx, Comte, Durkheim, Mamheim y otros, formularon teorías sobre el ocultamiento y el desvelamiento de la realidad social. Hegel afirmaba que la humanidad caminaba a ciegas respecto a sus propias realizaciones intelectuales porque carecía de autoconciencia histórica. A esto le llamó alienación. Marx colocó el problema en el plano económico y social: los trabajadores no tienen conciencia de la enajenación de su propio trabajo. No saben que producen la sociedad que los trata como un medio y nó como un fin.

Los autores mencionados colocaron sus teorías en el plano general de la sociedad pero no se ocuparon de la educación superior, salvo Emile Durkheim que a principios del siglo XX abre el camino a la sociología de la educación. Pasaron décadas hasta que en la segunda mitad del siglo comenzaran realmente los estudios sobre la universidad.

Entre mediados de 1969 y 1970 aparecieron movimientos estudiantiles y juveniles en China, la India, Estados Unidos, Europa Occidental y América Latina. Aparentemente no había mucha relación, salvo las conexiones ideológicas, entre la revolución cultural china y el pacifismo en el campus de California, o entre las protestas estudiantiles en Córdoba y la eclosión de "mayo 68" en París, entre los "naxalistas" de la India y el movimiento hippie. Detrás de todos estos movimientos había algunos factores comunes: el salto demográfico y generacional, la masividad del acceso a la educación superior y el extrañamiento de los jóvenes frente a la sociedad imperante.

El primer gran estudio comparativo sobre la evolución de las universidades se realizó en 1963 a través de la Comisión Robbins que organizó el Reino Unido y que dio lugar a doce volúmenes. Fue hacia 1960 que en América Latina aparece la propuesta de modernización universitaria de Rudolph Atcon y luego una serie de trabajos de la UDUAL, de Scherz 
García (Chile), de Darcy Ribeiro (Brasil) y de otros. La economía de la educación crece en este período y luego aparecen los trabajos de sociología de la educación que se consolidan entre 1970 y 1980. La pedagogía universitaria también aparece como un centro de interés en este momento.

Ahora comprendemos que se estaba generalizando la educación superior y que las sociedades estaban entrando en una economía con uso intensivo de la ciencia y la tecnología (la "economía de conocimiento intensivo" o el "capitalismo cognitivo", como se quiera llamar). Estaba naciendo el nuevo proletariado del conocimiento (DRUCKER, 1989). También podemos entender desde la perspectiva actual que los productores de conocimiento comienzan a reflexionar sobre sus propias prácticas: surge una epistemología crítica. La "reflexividad" se convierte en uno de los aspectos distintivos del nuevo modo de producción de conocimientos (GIBBONS, 1997).

En Europa la crisis universitaria de 1968 y la introducción de métodos de gestión modernos, obligaron a repensar las organizaciones vigentes. Nacen entonces los estudios económicos, administrativos y sociológicos sobre la educación superior. La UNESCO y la OCDE fueron pioneras en estos trabajos. Se crearon centros de estudios sobre la Educación Superior en Paris, Bucarest, Londres y otros lugares.

Estados Unidos figura entre los países que más larga tradición tienen en cuanto al estudio de la educación superior. El liderazgo mundial que comenzó a ejercer este país en materia universitaria a partir de la posguerra 1939-1945 lo llevó al estudio de su propio sistema y el de otros países. En América del Sur fueron los trabajos de la CEPAL los que incitaron, desde la teoría del desarrollo, a indagar sobre los impactos económicos y sociales de la educación.

La economía y la sociología de la educación comenzaron a realizar investigaciones sobre la importancia económica de la educación superior, sobre el acceso a la educación y la igualdad de oportunidades y otros temas. (Cabe recordar que uno de los primeros estudios sobre el impacto económico de la educación fue realizado en la Unión Soviética por Strumilin en 1921). Algunas cuestiones que habían sido planteadas en términos ideológicos o teóricos recibieron un sustento o una refutación científica. La afirmación ingenua de que la generalización de la educación generaba desarrollo ("teoría del capital humano", "teorías de la igualdad ante la educación") se encontraba con datos y experiencias que mostraban que si 
bien la formación de recursos humanos calificados incrementa el potencial de un país, esto no asegura que el país se desarrolle si no existen otras condiciones.

El trabajo de Pierre Bourdieu, La Reproducción (1970) mostraba que normalmente una sociedad tiende a reproducir las pautas dominantes. Esta tesis que hizo escuela e inspiró numerosos trabajos y discursos, condujo también a una simplificación pues se podía observar a nivel internacional que los sistemas de educación superior pueden ser también agentes de cambio. Esto había ocurrido en el Japón de fines del siglo XIX y ocurre en el siglo XX con casos como los de Corea, China, India. Los estudios comparativos se volvieron cruciales para controlar las afirmaciones sobre la función social de la educación. En todo caso, mostraban que la educación superior al mismo tiempo que reproduce una cultura también produce las condiciones de una nueva sociedad.

\section{El florecimiento de los estudios sobre la educación superior}

La convergencia de distintas preocupaciones sobre la educación superior (la masificación a partir del acceso generalizado de los jóvenes de entre 17-24 años, la vinculación entre educación y desarrollo, la importancia de la investigación) llevó a estudios históricos, sociológicos, económicos, políticos y pedagógicos que crearon un nuevo panorama sobre el conocimiento de la educación superior hacia comienzos del siglo XXI.

En efecto, si uno analiza la producción bibliográfica en América del Sur sobre la universidad durante los últimos veinte años podrá apreciar la explosión de publicaciones e informes. Sin contar con los coloquios y congresos relacionados con el tema. Solo en Argentina podemos encontrar que si en 1986 había dos estudios importantes sobre el sistema universitario hacia el 2007 tenemos más de treinta títulos. A su vez, en 1986 no encontramos ningún programa de posgrado sobre la educación superior en Argentina mientras que en 2007 tenemos más de veinte (que se encuentran vinculados a la Red Argentina de Posgrados en Educación Superior).

La reflexión sobre la universidad se ha incrementado. Este fenómeno tiene que ver con varios factores: el surgimiento de la economía cognitiva, la informatización de la sociedad, la globalización, los estudios de epistemología crítica y la aparición de las políticas de certificación de calidad (en los productos de la economía, en los servicios y en la educación). 
Esta contextualización debiera permitirnos superar algunos clichés sobre el significado de las políticas de evaluación universitaria.

En lo que se denomina "la sociedad del conocimiento", podemos identificar por un lado el incremento de la "reflexión" sobre la producción de los saberes (algo que a nivel institucional se refleja en el desarrollo de los sistemas de evaluación) y por otro lado, la necesidad de sistematizar la formación de los administradores de la actividad científica y educativa (lo que en las empresas dio lugar al "knowledge management").

El trabajo de Michael Gibbons y otros, "La nueva producción del conocimiento" (1997) analiza la dinámica de la ciencia y la investigación en las sociedades contemporáneas. Otros estudios que le siguen, como "ReThinking Science" (NOWOTNY, 2003), muestran que ahora la cuestión de la educación superior se plantea a un nivel más profundo: el de la relación entre la producción y la distribución de conocimientos en las sociedades avanzadas. Y esto inevitablemente conduce a plantear cuestiones epistemológicas de todo tipo.

Entre las características que Michael Gibbons y colaboradores atribuyen al modo emergente de producción de conocimientos podemos mencionar: la transdisciplinariedad (versus la monodisciplinariedad de los programas académicos); la heterogeneidad (versus homogeneidad o univocidad de las formaciones profesionales); la contextualización (versus la endogamia disciplinar); el conocimiento orientado a la resolución de problemas (versus el cientificismo y el academicismo); la interactividad horizontal entre los actores (versus la verticalidad); el conocimiento extendido a la sociedad (versus el elitismo de los expertos), la mayor responsabilidad social (versus el individualismo académico); la mayor reflexividad (versus la cultura científica sin conciencia de sus funciones sociales). Cada uno de estos aspectos está vinculado a debates epistemológicos y sociológicos cuyo inicio podríamos situar en 1961 con la publicación del libro de Thomas Kuhn, "La estructura de las revoluciones científicas".

No podemos dejar de mencionar también el crecimiento de los trabajos en ciencias cognitivas que nacieron hacia 1956 como convergencias entre el conductismo, la psicología cognitiva, la teoría de sistemas, la informática, la inteligencia artificial, la cibernética y las neurociencias. Si bien estas y otras teorías tuvieron desarrollos paralelos y a veces aislados o incompatibles entre sí, lo que hoy se puede apreciar es el intento de pensar todos estos procesos de manera transdisciplinaria. (GARDNER, 1987). 
Este rastreo genealógico relacionado con el estudio de la educación superior también nos ilumina sobre el sentido de la evaluación institucional. Por un lado, aparece como un momento más del proceso por el cual las sociedades contemporáneas tienden a producirse a través del conocimiento y del autoconocimiento. Por otro lado, aparece como un mecanismo de control de calidad que aplicado a la educación superior tiene que cumplir con ciertas exigencias epistemológicas. Acontece que ni las agencias de evaluación, ni las universidades, ni los estudios de la educación superior suelen conectar todas las dimensiones epistemológicas, cognitivas, educativas, sociales o económicas que justifican la cultura de la evaluación.

\section{Complejidad y transdisciplinariedad}

Ahora sabemos que las organizaciones universitarias son las más complejas que pueden existir. O sea, resultan más complejas que los partidos políticos, las empresas, las iglesias, los bancos, las fuerzas armadas, los sindicatos. Desde la Edad Media las universidades reúnen en sí mismas capacidades doctrinales como la Iglesia, competencias judiciales, atributos corporativos, vinculaciones con el poder político y por supuesto incumbencias pedagógicas y científicas. (PERKINS, 1973).

Reconocer a la educación superior como un objeto complejo es un primer paso indispensable para abordar epistemológicamente la cuestión. Un corolario de la complejidad es la transdisciplinariedad que exige el tema.

A la complejidad del objeto y a la necesidad de un abordaje transdisciplinario hay que agregar el enfoque cognitivo. Hasta ahora, en efecto, se ha analizado la educación superior como sistema, o como organización, o como institución o como centro de enseñanza-aprendizaje. Por supuesto, se trata de enfoques pertinentes de acuerdo con la naturaleza del objeto. Pero, curiosamente, se ha prestado poca atención al hecho de que la educación superior supone teorías del conocimiento y criterios de justificación de las mismas. Este sería el núcleo epistemológico del objeto.

¿Qué podría cambiar mirando a la educación superior como un proceso de producción, de validación y de distribución de conocimientos?. El enfoque dominante nos dice que la educación superior se ocupa principalmente de formar profesionales para satisfacer las demandas de la sociedad. Este supuesto refuerza el peso de las formaciones profesionalistas, de la 
cultura monodisciplinar y de la influencia de los colegios profesionales sobre la universidad. El currículo de cada Facultad y a veces el gobierno de las mismas depende de la cultura profesionalista.

¿Qué pasa si en lugar de mirar las cosas desde el punto de vista del mercado profesional lo hacemos desde el punto de vista del mercado del conocimiento?. Podríamos descubrir que sobran profesionales, pero faltan múltiples aplicaciones de conocimientos en la sociedad. (Sobran abogados y falla la administración de justicia, sobran médicos y el sistema de salud es deficiente, sobran arquitectos y faltan viviendas para el pueblo, etc.).

Durante mucho tiempo la transmisión de conocimientos venía en envases codificados por las disciplinas y profesiones. Ahora tenemos problemas que requieren competencias diversas. Por ejemplo: los problemas urbanos, de la seguridad, de la violencia, de la contaminación ambiental, del recalentamiento global, del desarrollo, de la informatización de la sociedad, etc. ¿Quién debe intervenir en casos de violencia escolar? ¿Los pedagogos, los psicólogos, los sociólogos, los administradores de la educación, los agentes de seguridad?. Ocurre que en las facultades respectivas ni los psicólogos, ni los pedagogos, ni los sociólogos, ni los agentes de seguridad, tienen en su currículo un programa para investigar y actuar de manera conjunta sobre la violencia escolar. De hecho, son las autoridades educativas las que deciden formar equipos interdisciplinarios para ver como afrontar la cuestión.

Es que la clave está en la naturaleza de los problemas (que son complejos) y en las políticas para resolverlos (que tienen que ser interdisciplinarios e interinstitucionales). En los estudios sobre el nuevo modo de producción de conocimientos Michael Gibbons y otros autores subrayan que en el campo de la actividad científica ya no pueden reproducirse las pautas de los trabajos monodisciplinarios si queremos resolver los problemas de la sociedad. Sabemos por constatación empírica que cerca del $60 \%$ de los ejecutivos de empresas y funcionarios superiores del Estado son personas que tuvieron inicialmente una formación distinta a la de sus competencias actuales.

Si miramos las cosas desde el punto de vista de los procesos del conocimiento necesitamos reconocer en su dimensión genética como intervienen la realidad, la subjetividad, el lenguaje, las comunicaciones sociales y otros factores en la construcción de la inteligencia humana a través de los aprendizajes. Pero gran parte de la enseñanza universitaria, entre el $80-90 \%$, es informativa y descriptiva. Los sistemas de información 
están reemplazando las funciones de la memoria. No se tiene en cuenta que con las nuevas tecnologías de la información tienen que reforzarse las competencias cognitivas, el aprender a pensar, el aprender a aplicar los conocimientos.

Los análisis institucionales, pedagógicos, organizacionales y políticos predominan en los estudios sobre la educación superior. Si tomamos en cuenta las ponencias presentadas en los congresos sobre educación superior tendremos un $80 \%$ de estudios didácticos y pedagógicos, contra un $20 \%$ de estudios institucionales y de gestión. Pero el modo de producción y de transmisión de conocimientos está cambiando de tal modo que tanto el currículo (disciplinar, profesional), como las estructuras de enseñanza (presencial, compartimentada) tienden a quedar desfasados. Es por esta razón, entre otras, que la reforma de la educación superior en Europa (a partir de los Acuerdos de Bologna, 1999) tienden a reducir la transmisión de contenidos privilegiando la formación por competencias (el nuevo espacio europeo del conocimiento).

Hemos señalado así la centralidad que van a tener los problemas epistemológicos y cognitivos en los nuevos contextos de la educación superior. Pero el estudio de las prácticas vigentes (la enseñanza en el aula, los problemas de gestión, de organización, de políticas) no dejará de tener relevancia. Ahora bien, para analizar muchas de estas cuestiones se necesitan instrumentos de análisis de mayor cientificidad. Muchas de las observaciones que se hacen sobre las universidades son triviales. Cuando un investigador observa las prácticas universitarias descubre que los discursos de los actores no se corresponden con la experiencia. A veces los profesores ignoran las condiciones de comunicación y el aula se convierte en un sistema sinfeed-back donde reina la ley del silencio de acuerdo a la teoría de la información.

Así como la formación de los profesores y de los administradores universitarios tiende a profesionalizarse, del mismo modo la observación del mundo universitario tiende a ser más científica. Lo que resulta coherente con el discurso científico que las universidades intentan aplicar en todos los otros terrenos. No hay razón para pensar que el estudio de las prácticas universitarias merezca menos exigencias científicas que el estudio de los comportamientos sociales o de los procesos económicos o de los fenómenos de contaminación ambiental.

Las universidades producen estadísticas, informes institucionales y evaluaciones que se aprovechan muy marginalmente en estudios científi- 
cos sobre la universidad misma. Las evaluaciones institucionales pueden ser una gran fuente de estudio siempre y cuando las observaciones estén organizadas del mismo modo que se hace en un laboratorio o en un trabajo científico. Sin duda, la evaluación institucional apela a un juicio de valor de un experto y a una serie de interpretaciones de los actores. Sin desdeñar la pluralidad de juicios (que forman parte del "círculo hermeneútico" de toda evaluación) no podemos opinar sobre temas tan críticos como el fracaso académico o la crisis de los sistemas de gobierno sin elaborar hipótesis pertinentes sobre los fenómenos que estamos observando. En el campo de la salud las estadísticas hospitalarias y los estudios sociales sirvieron muchas veces para verificar o refutar hipótesis sobre las enfermedades. Pero esto requiere crear sistemas de observación y de contrastación de hipótesis adecuados.

La observación empírica, el análisis estadístico, el análisis pedagógico, sociológico, económico o antropológico, el estudio de las políticas, del sistema normativo o de gobierno, son todos enfoques posibles para estudiar la educación superior. Pero cuando parece que agotamos el análisis sincrónico de un aspecto de la universidad encontraremos que hace falta una perspectiva histórica. En efecto, muchas de las cosas que ahora consideramos mejorables tal vez tiendan a transformarse en el futuro. La mirada anticipatoria, prospectiva, es otro paso decisivo para analizar y evaluar las universidades. ¿Cuánto va a crecer la enseñanza virtual? ¿Qué perfiles científicos y pedagógicos se necesitarán en diez o veinte años para ser un buen profesor universitario? ¿Cómo impactará en las carreras de los estudiantes la movilidad creciente de los mismos entre distintos países? ¿Hasta dónde podemos informatizar el sistema de gestión de las universidades?.

Por otro lado, no debemos olvidar que el campo de la educación superior está sujeto a valores, modelos culturales, filosofías e ideologías que pugnan por imponerse o que se imponen de manera inconsciente. Al registrar todos estos aspectos tal vez tomemos conciencia de que el objeto es inagotable. Y lo es. Como en otras dimensiones donde interviene el pensamiento y la acción humana.

Necesitamos el estudio objetivo con todos los métodos que nos permitan llegar a un conocimiento verosímil, como diría Popper. Esto nos ayudaría mucho a tomar decisiones eficaces. El grado de desconocimiento con el que se administran en la actualidad las universidades no puede menos que conducirnos a errores inevitables. Si navegamos sin rumbo 
cualquier destino es posible. Una cierta responsabilidad moral nos indica que no podemos desperdiciar recursos escasos trabajando de manera ineficiente. Sobre todo, cuando se trata del futuro de nuestros países y de miles de jóvenes que dependen de un proyecto adecuado para integrarse en la sociedad.

El conocimiento científico es una condición para tomar decisiones correctas. Saber para poder. Pero en última instancia la educación superior no nos interesa solo desde el punto de vista de su eficiencia académica o de su buena administración. Una universidad está ligada a un modelo de desarrollo del cual dependen también las condiciones de progreso de los individuos y grupos sociales. La gestión del conocimiento tiene un aspecto cognitivo y otro aspecto político. Este aspecto político en el sentido más profundo comporta una teoría de la acción y una idea moral de la sociedad. El estudio de la educación superior por lo tanto desemboca en una teoría general de la universidad vinculada al devenir de la sociedad.

\section{Epistemología y evaluación de la educación superior}

Parece lógico reclamar que el estudio de la educación superior se someta a criterios epistemológicos. También parece lógico pedir que las agencias de evaluación que deben dictaminar sobre la pertinencia de los programas académicos trabajen con métodos científicos. Ya en 1967 Edward Suchman preconizaba el método científico de la evaluación en su libro "Evaluative Research: Principles and Practice in Public Service and Social Action Programs." (STUFFLEBEAM; SHINKFIELD, 1987).

Por supuesto, existen criterios políticos, culturales o éticos que trascienden la cientificidad de la educación superior. Por eso, la evaluación puede tener también distintos objetivos. El problema es que la construcción de una idea de la universidad se juega en el conocimiento y éste se rige por reglas que legitiman los saberes y las competencias de los individuos que analizan o administran la universidad. Seguramente, la reforma actual de la Educación Superior en Europa tiene que tomar en cuenta factores históricos, culturales o políticos, pero en última instancia el modelo pedagógico y organizacional del nuevo espacio europeo del conocimiento tiene que ajustarse a criterios pedagógicos y racionales conformes con la cultura académica.

En la era de la sociedad del conocimiento parece lógico recurrir a la epistemología para fundamentar las políticas y la gestión universitaria. Por 
imperio de la disociación que todavía padecemos los epistemólogos se interesan poco por los análisis institucionales, que quedan en manos de las ciencias administrativas, y los expertos en gestión se interesan poco en cuestiones epistemológicas. Es interesante notar que la "gestión del conocimiento" preconizada por Nonaka y Takeuchi propone superar esta disociación en el gerenciamiento organizacional (TAKEUCHI; NONAKA, 2004).

Si lo que tenemos que analizar es una organización que produce, organiza, distribuye y aplica conocimientos no parece coherente ignorar que hay una disciplina que se ocupa de los procesos cognitivos y de la legitimación de los saberes. Esa es la epistemología. A su vez, la epistemología no puede ignorar las ciencias cognitivas, la sociología del conocimiento, el análisis institucional, la teoría de la educación, la economía del saber y otras disciplinas conexas.

El modo de producción y de transmisión de conocimientos se está transformando rápidamente dejando a las estructuras académicas que se inventaron en otros contextos en situación vulnerable. Algunas de esas estructuras se verán en poco tiempo como la arqueología del saber o como fósiles académicos. Entre las tendencias al cambio y lo que merece ser conservado también es preciso realizar un balance. Uno de los desafíos más grande de la gestión del conocimiento en las universidades tiene que ver con eso: con la elaboración de criterios para descartar lo que es perecedero y para conservar lo que tiene un valor perenne, o sea, lo que forma parte del patrimonio de la cultura universal.

La epistemología es la disciplina pertinente para ayudarnos a comprender los contextos de descubrimiento, de justificación y de aplicación de los conocimientos. Lo cual debería ser un requisito de todo administrador universitario. Normalmente la gestión universitaria se focaliza en las estructuras, el manejo del presupuesto, el gobierno, la gestión de personal o el funcionamiento de las carreras y cursos. Evidentemente, hay que ocuparse de estas cosas. Pero no podemos olvidar que la materia prima y la sustancia de lo que hacemos en la universidad es producir y transferir conocimientos cuyos impactos deberíamos ser capaces de prever.

El estudio de los contextos de descubrimiento nos permite reconstruir las condiciones bajos las cuales aparecen nuevas fronteras del conocimiento y nuevos paradigmas que nos obligan a redefinir los planes de estudios, las carreras y las políticas de investigación. 
El análisis de los contextos de justificación nos ayuda a mejorar la calidad de la enseñanza y los criterios de evaluación de los proyectos de investigación. A este nivel es donde se plantean las cuestiones de pertinencia teórica de los programas de investigación que todos los especialistas consideran como un criterio básico para las políticas científicas.

El estudio de los contextos de aplicación nos remite al tema de la pertinencia social de la actividad académica y científica. ¿Qué problemas estamos tendiendo a resolver?. ¿Qué demanda de recursos humanos calificados y de conocimientos científicos estamos atendiendo?. ¿Cómo nos insertamos en las nuevas condiciones de innovación tecnológica?

Es evidente que estas cuestiones no se pueden atender solamente con visiones aisladas. Por eso creemos que es conveniente tomar en cuenta las investigaciones sobre la educación superior que analizan la evolución de las universidades, de la ciencia, de las innovaciones tecnológicas, de las profesiones y de la sociedad.

Desde este punto de vista afirmamos que el futuro de la educación superior en América del Sur, y en parte el futuro mismo del modelo de desarrollo, depende de la capacidad de los dirigentes para convertir el conocimiento disponible sobre la enseñanza y la investigación universitaria en políticas institucionales que permitan dar un salto cualitativo en el progreso de la región.

\section{REFERÊNCIAS}

BECHER, Tony. Tribus y territorios académicos. Barcelona: Gedisa, 2001. BOURDIEU, P.; PASSERON, J.C. La reproduction. Paris: Minuit, 1970. CLARK, Burton. El sistema de educación superior. México: Nueva Imagen, 1983.

CLARK, Burton. The higer education system. California: University of Calofornia Press, 1986.

DEBELLE, Jean. La recherche sur l'université facteur de son évolution. Bruxelles: Vie Ouvrière, 1986.

DIAS SOBRINHO, José; RISTOFF, Dilvo (Orgs.). Universidade deconstruída. Florianópolis: Insular, 2000.

DIAS SOBRINHO, José. Universidade e avaliação, entre a ética e o mercado. Florianópolis: Insular, 2002. 
DRUCKER, Peter. Las nuevas realidades. Buenos Aires : Sudamericana, 1989.

FERNANDEZ LAMARRA, N. Educación superior y calidad en América Latina y Argentina. Los procesos de evaluación y acreditación. Buenos Aires: Eduntref, 2007.

GIBBONS, M. et al. La nueva producción del conocimiento. Barcelona: Pomares-Corregidor, 1997.

GARDNER, H. La nueva ciencia de la mente. Historia de la revolución cognitiva. Barcelona: Paidós, 1987.

KELLEY, D. (Ed.). History and the disciplines. The reclasification of knowledge in early modern Europe. Rochester: The University of Rochester Press, 1997.

NOWOTnY, H.; SCOTT, P.; GIBBOnS, M. Re-Thinking Science. Knowledge and the Public in an Age of Uncertainty. Oxford: Blackwell, 2001. PEREZ LINDO, Augusto. Universidad, política y sociedad. Buenos Aires: Eudeba 1985.

PEREZ LINDO, Augusto. Políticas del conocimiento, educación superior y desarrollo. Buenos Aires: Biblos, 1998.

PEREZ LINDO, Augusto. Universidad, conocimiento y reconstrucción nacional, Buenos Aires: Biblos, 2003.

PEREZ LINDO, Augusto et al. Gestión del conocimiento. Un nuevo enfoque aplicable a las organizaciones y a la universidad. Buenos Aires: Norma, 2005. PERKINS, James. The university as an organization. New York: McGraw Hill, 1973.

QUINTAS, Guillermo (Ed.). Reforma y evaluación de la universidad. Valencia: Universitat de Valencia, 1996.

RIBEIRO, Darcy. La universidad necesaria. México: UNAM, 1982.

ROTHBLATT, S.; BJORN, W. (Comp.). La universidad europea y americana desde 1800. Barcelona: Pomares - Corregidor, 1996.

STUFFLEBEAM, D.; SHINKFIELD, A. Evaluación sistemática. Guía teórica y práctica. Barcelona: Paidós, 1987.

TAKEUCHI, H.; NONAKA, I. Hitotsubashi on knowledge management. Singapur: Wiley \& Son, , 2004.

TUNNERMANN BERNHEIM, Carlos. Estudios sobre la teoría de la universidad. San José de Costa Rica: Editorial Universitaria Centroamericana, 1983. 